\title{
Compressive Sensing Based Bayesian Sparse Channel Estimation for OFDM Communication Systems: High Performance and Low Complexity
}

\author{
Guan Gui, ${ }^{1}$ Li Xu, ${ }^{2}$ Lin Shan, ${ }^{3}$ and Fumiyuki Adachi ${ }^{1}$ \\ ${ }^{1}$ Department of Communications Engineering, Graduate School of Engineering, Tohoku University, Sendai 980-8579, Japan \\ ${ }^{2}$ Faculty of Systems Science and Technology, Akita Prefectural University, Akita 015-0055, Japan \\ ${ }^{3}$ Wireless Network Research Institute, National Institute of Information and Communications Technology (NICT), \\ Yokosuka 239-0847, Japan
}

Correspondence should be addressed to Guan Gui; gui@mobile.ecei.tohoku.ac.jp

Received 12 February 2014; Accepted 24 March 2014; Published 10 April 2014

Academic Editors: J. Lloret and Y. Mao

Copyright (C) 2014 Guan Gui et al. This is an open access article distributed under the Creative Commons Attribution License, which permits unrestricted use, distribution, and reproduction in any medium, provided the original work is properly cited.

\begin{abstract}
In orthogonal frequency division modulation (OFDM) communication systems, channel state information (CSI) is required at receiver due to the fact that frequency-selective fading channel leads to disgusting intersymbol interference (ISI) over data transmission. Broadband channel model is often described by very few dominant channel taps and they can be probed by compressive sensing based sparse channel estimation (SCE) methods, for example, orthogonal matching pursuit algorithm, which can take the advantage of sparse structure effectively in the channel as for prior information. However, these developed methods are vulnerable to both noise interference and column coherence of training signal matrix. In other words, the primary objective of these conventional methods is to catch the dominant channel taps without a report of posterior channel uncertainty. To improve the estimation performance, we proposed a compressive sensing based Bayesian sparse channel estimation (BSCE) method which cannot only exploit the channel sparsity but also mitigate the unexpected channel uncertainty without scarifying any computational complexity. The proposed method can reveal potential ambiguity among multiple channel estimators that are ambiguous due to observation noise or correlation interference among columns in the training matrix. Computer simulations show that proposed method can improve the estimation performance when comparing with conventional SCE methods.
\end{abstract}

\section{Introduction}

In broadband wireless communication systems using orthogonal frequency division modulation (OFDM), frequencyselective fading is incurred by the reflection, diffraction, and scattering of the transmitted signals due to the buildings, large moving vehicles, mountains, and so forth. Such fading phenomenon distorts received signals and poses critical challenges in the design of communication systems for highrate and high-mobility wireless communication applications. Hence, accurate channel estimation becomes a fundamental problem of such communication systems. In last several years, various linear estimation methods have been proposed based on the assumption of rich multipath channel model. However, recently, a lot of physical channel measurements verified that the channel taps exhibit sparse distribution [1-3] due to the broadband signal transmission. A typical example of sparse multipath channel is shown in Figure 1 where the length is 100 while the number of nonzero taps is 5 only. Note that different broadband transmission may incur different channel structures in wireless communication systems as shown in Table 1.

To improve the estimation performance, extra sparse structure information can be exploited as prior information. Thanks to the development of compressive sensing $[4,5]$, many sparse channel estimation (CCE) methods have been proposed for exploiting the channel sparsity. In [6], orthogonal matching pursuit (OMP) algorithm with application to sparse multipath channel estimation in the OFDM systems has been proposed. In $[7,8]$, sparse channel estimation methods have been proposed using compressive sampling 
TABLE 1: Channel structures in different mobile communication systems.

\begin{tabular}{lccc}
\hline Generations of mobile communication systems [21] & $\begin{array}{c}2 \mathrm{G} \text { cellular } \\
\text { (IS-95) }\end{array}$ & $\begin{array}{c}\text { 3G cellular } \\
\text { (WCDMA) }\end{array}$ & $\begin{array}{c}4 \mathrm{G} / 5 \mathrm{G} \text { cellular } \\
\text { (LTE-Advanced ) }\end{array}$ \\
\hline Transmission bandwidth & $1.23 \mathrm{MHz}$ & $10 \mathrm{MHz}$ & $20 \mathrm{MHz} 100 \mathrm{MHz}$ \\
Time-delay spread (for example) & $0.5 \mu \mathrm{s}$ & $0.5 \mu s$ & $0.5 \mu s$ \\
Sampling channel length & 1 & 10 & $20 \sim 100$ \\
Number of nonzero taps & 1 & 4 & $2 \sim 10$ \\
Channel structure model & Dense & Approximate sparse & Sparse \\
\hline
\end{tabular}

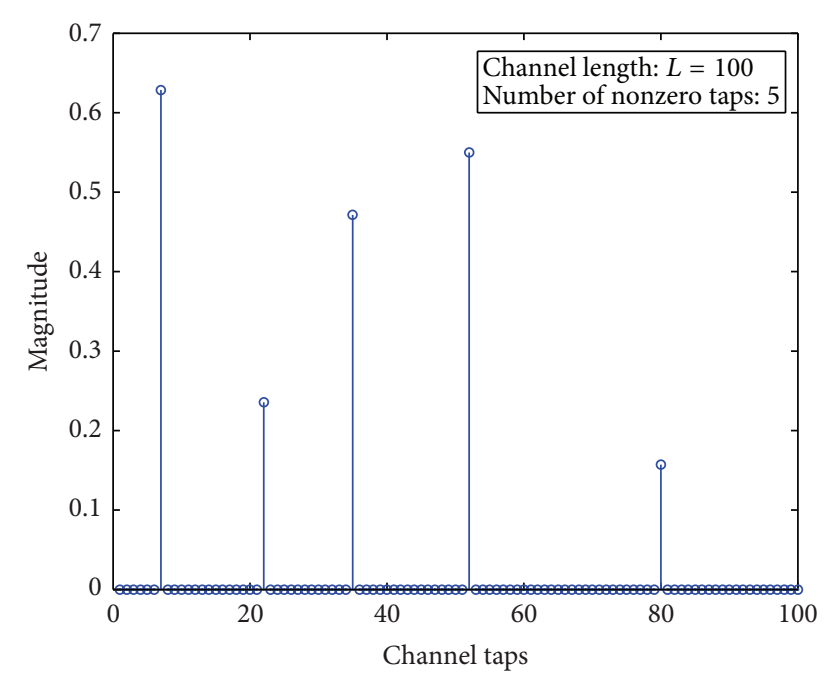

FIGURE 1: A typical example of sparse multipath channel.

matching pursuit (CoSaMP) algorithm [9] in frequencyselective and doubly-selective channel fading communication systems. In [10], to further reduce the computational complexity, sparse channel estimation using smooth $\ell_{0^{-}}$ norm (SL0) algorithm [11] has been proposed. Compared to traditional linear methods, sparse channel estimation methods have two obvious advantages: spectral efficiency and lower performance bound. For one thing, improving the spectral efficiency by utilizing less training sequence can achieve the same estimation performance as linear methods. For another, the lower performance bound can be obtained by exploiting channel sparsity due to the fact that less active channel freedom of degree is acquired [12].

Conventional sparse channel estimation methods have a cardinal objective that try to probe the dominant channel taps as accurate as possible, while these methods neglect the posterior information report from additive noise received signal. These proposed channel estimation methods are termed as model selection or basis selection. Unfortunately, their estimation performances are often degraded due to the neglecting channel model uncertainty [13]. To mitigate the unexpected model uncertainty, Bayesian compressive sensing (BCS) [14] and a slight improved Bayesian compressive sensing using Laplace priors (BCS-LAP) [15] could be adopted for estimating sparse channel. The estimation performance could be improved effectively but at the cost of high computational complexity when comparing with existing simple algorithms (e.g., OMP [6] and SL0 [11]). Hence, it is impractical to employ this algorithm in real communication systems.

Unlike these aforementioned methods, in this paper, we propose an improved Bayesian sparse channel estimation (BSCE) method while its computational complexity is comparable with OMP and SL0. Our proposed Bayesian channel method can be divided into two steps: position detection of dominant channel taps and channel estimation using minimum mean square error (MMSE). In general, our proposed Bayesian estimation method provides model uncertainty which reveals uncertainty among multiple possible position sets of dominant channel taps that are ambiguous due to observation noise or correlation among columns in the training matrix. Furthermore, the complexity of the proposed method is relatively lower due to its smaller search space when compared to conventional methods. Simulation results are given to verify two folds: performance and complexity. Note that estimation performance is evaluated by two metrics: mean-square-error (MSE) and bit-error rate (BER), while computational complexity is measured coarsely by CPU time of computer.

The remainder of this paper is organized as follows. An OFDM system model is described and problem formulation is given in Section 2. In Section 3, the BSCE method is proposed in OFDM systems. Computer simulation results are given in Section 4 in order to evaluate and compare performance of the BSCE method with conventional methods. Finally, we conclude the paper in Section 5.

Notation 1. Throughout the paper, matrices and vectors are represented by boldface upper case letters (i.e., $\mathbf{X}$ ) and boldface lower case letters (i.e., $\mathbf{x}$ ), respectively; the superscripts $(\cdot)^{T},(\cdot)^{H},(\cdot)^{-1}$, and $\operatorname{diag}(\cdot)$ denote the transpose, the Hermitian transpose, and the inverse and diagonal operators, respectively; $E\{\cdot\}$ denotes the expectation operator; $\|\mathbf{h}\|_{0}$ is the $\ell_{0}$-norm operator that counts the number of nonzero taps in $\mathbf{h}$; and $\|\mathbf{h}\|_{p}$ stands for the $\ell_{p}$-norm operator which is computed by $\|\mathbf{h}\|_{p}=\left(\Sigma_{l}\left|h_{l}\right|^{p}\right)^{1 / p}$, where $p \in\{1,2\}$ is considered in this paper.

\section{System Model and Problem Formulation}

Consider a frequency-selective multipath channel whose impulse response is given by

$$
\mathbf{h}=\sum_{l=0}^{L-1} h_{l} \delta\left(\tau-\tau_{l}\right),
$$


where $L$ is the number of multipaths and $h_{l}$ and $\tau_{l}$ are the (complex) channel gain and the delay spread, respectively, of path $l$ at time $t$. Hence, the $L$-length discrete channel vector can be written as $\mathbf{h}=\left[h_{0}, h_{1}, \ldots, h_{L-1}\right]^{T}$. Let the OFDM system use size- $N$ discrete Fourier transform (DFT), and its number of pilot subcarriers is $N_{p}$. To avoid intersymbol interference (ISI), we assume that the length $N_{g}$ of the zeropadding cyclic prefix (CP) in the OFDM symbols is larger than maximum delay spread $\tau_{\max }$, where $\tau_{\max } \geq \tau_{l}, l=$ $0,1, \ldots, L-1$. Suppose that $\bar{X}(i)$ denote $i$ th subcarrier in an OFDM symbol, where $i=0,1, \ldots, N-1$. If the coherence time of the channel is much larger than the OFDM symbol duration $T$, then the channel can be considered quasistatic over an OFDM symbol. Let $\bar{y}$ be the vector of received signal samples in one OFDM symbol after DFT; then

$$
\overline{\mathbf{y}}=\overline{\mathbf{X h}}+\overline{\mathbf{z}}=\overline{\mathbf{X}} \mathbf{F h}+\overline{\mathbf{z}}=\mathbf{X h}+\mathbf{z},
$$

where $\overline{\mathbf{X}}=\operatorname{diag}\{X(0), X(1), \ldots, X(N-1)\}$ denotes diagonal subcarrier matrix, $\overline{\mathbf{h}}$ is the channel frequency response (CFR) in frequency-domain, and $\overline{\mathbf{z}}$ is assumed to be additive white Gaussian noise (AWGN) with variance $\sigma^{2} . \mathbf{F}$ is an $N \times L$ partial DFT matrix with its $k$ th row which is easily given by $1 / \sqrt{N}\left[0, e^{-j 2 \pi k / N}, \ldots, e^{-j 2 \pi k(L-1) / N}\right]$ and $\mathbf{X}=\overline{\mathbf{X}} \mathbf{F}=$ $\left[\mathbf{x}_{0}, \ldots, \mathbf{x}_{l}, \ldots, \mathbf{x}_{L-1}\right]$ denotes an $N \times L$ equivalent timedomain signal matrix. In addition, $\mathbf{h}=\left[h_{0}, h_{1}, \ldots, h_{L-1}\right]^{T}$ denotes a $L \times 1$ time-domain channel vector. Since $\overline{\mathbf{h}}=\mathbf{F h}$, hence, the frequency-domain channel impulse response $\overline{\mathbf{h}}$ lies in the time-delay spread domain.

Assume that a binary random vector $\mathbf{g}=$ $\left[g_{0}, g_{1}, \ldots, g_{L-1}\right]^{T}$ denotes a taps' position indicator of sparse channel vector $\mathbf{h}$ which is generated from a Gaussian mixture density (GMD) function as

$$
\{\mathbf{h} \mid \mathbf{g}\} \sim \mathscr{C} \mathscr{N}(\mathbf{0}, \mathbf{R}(\mathbf{g})),
$$

where the covariance matrix $\mathbf{R}(\mathbf{g})$ is determined by position indicator $\mathbf{g}$. For a better understanding, we take $\mathbf{R}(\mathbf{g})$ to be diagonal element with $[\mathbf{R}(\mathbf{g})]_{l l}=\sigma_{l}^{2}=\sigma_{1}^{2}$ for $l=$ $0,1, \ldots, L-1$, implying that $\left\{h_{l} \mid g_{l}\right\}_{l=0}^{L-1}$ are independent with Gaussian distribution $\left\{h_{l} \mid g_{l}\right\} \sim \mathscr{C} \mathscr{N}\left(0, \sigma_{1}^{2}\right)$. Assume that the position indices $\left\{g_{l}\right\}_{l=0}^{L-1}$ are satisfied Bernoulli distribution with probability $p_{1, l}$; then the probability of nonzero and zero channel taps of channel vector $\mathbf{h}$ can be written as

$$
\begin{gathered}
h_{l} \neq 0 \Longleftarrow \operatorname{Pr}\left\{g_{l}=1\right\}=p_{1, l}, \\
h_{l}=0 \Longleftarrow \operatorname{Pr}\left\{g_{l}=0\right\}=1-p_{1, l},
\end{gathered}
$$

for $l=0,1, \ldots, L-1$. According to (4), one can easily find $\|\mathbf{h}\|_{0}=\|\mathbf{g}\|_{1}$. In real communication systems, broadband channels are often described by sparse models $[16,17]$. Hence, we choose $\sigma_{0}^{2}=\operatorname{var}\left\{h_{0} \mid g_{0}\right\}=0$ and $p_{1}=\sum_{l=0}^{L-1} p_{1, l} \ll$ 1 , so that $\mathbf{h}$ has relatively few dominant channel taps. In other words, sparseness of channel vector $\mathbf{h}$ depends on the probability $p_{1}$ as shown in Figure 2. Smaller probability $p_{1}$ implies sparser channel vector $\mathbf{h}$ and vice versa.

The research objective of this paper is to estimate the sparse channel vector $\mathbf{h}$ using received signal vector $\mathbf{y}$ and

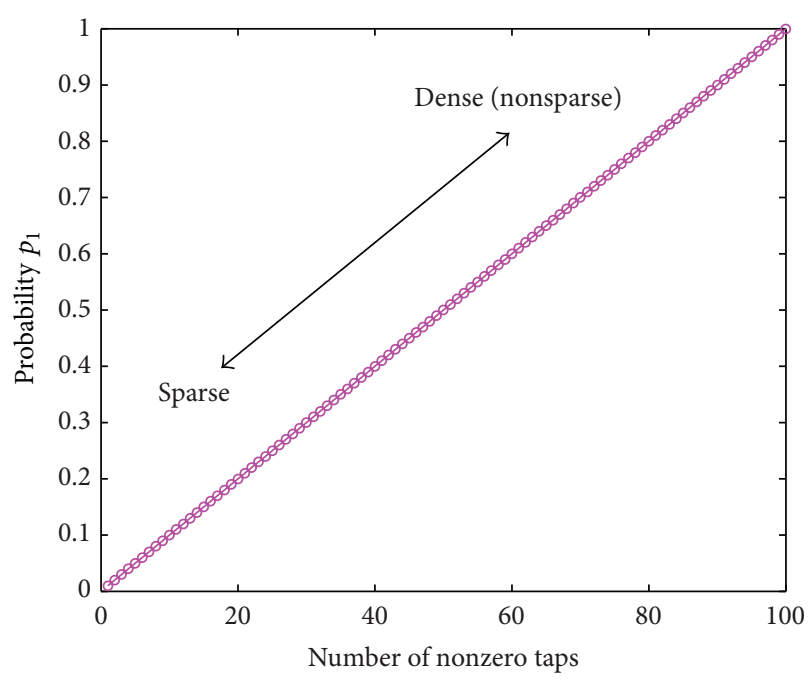

FIGURE 2: Sparseness of channel vector $\mathbf{h}$ depends on the probability $p_{1}$.

training signal matrix $\mathbf{X}$. Hence, the system model can be assumed to satisfy distribution as

$$
\begin{aligned}
{\left[\begin{array}{l}
\mathbf{y} \\
\mathbf{h}
\end{array}\right] } & \mid \mathbf{g} \sim \mathscr{C} \mathcal{N}\left(\mathbf{0},\left[\begin{array}{cc}
\mathbf{C}(\mathbf{g}) & \mathbf{X R}(\mathbf{g}) \\
\mathbf{R}(\mathbf{g}) \mathbf{X}^{T} & \mathbf{R}(\mathbf{g})
\end{array}\right]\right) \\
& =\mathscr{C} \mathscr{N}\left(\mathbf{0},\left[\begin{array}{cc}
\mathbf{C}(\mathbf{g}) & \sigma_{1}^{2} \mathbf{X I}_{L} \\
\sigma_{1}^{2} \mathbf{I}_{L} \mathbf{X}^{T} & \sigma_{1}^{2} \mathbf{I}_{L}
\end{array}\right]\right),
\end{aligned}
$$

where $\mathbf{C}(\mathbf{g}):=\mathbf{X R}(\mathbf{g}) \mathbf{X}^{T}+\sigma_{n}^{2} \mathbf{I}_{N}=\sigma_{1}^{2} \mathbf{X I}_{L} \mathbf{X}^{T}+\sigma^{2} \mathbf{I}_{N}$ is the covariance matrix of $\left\{\begin{array}{l|l}\mathbf{y} & \mathbf{g}\end{array}\right\}$. That is, $\left\{\begin{array}{l|l}\mathbf{y} & \mathbf{g}\end{array}\right\} \sim$ $\mathscr{C} \mathcal{N}\left(\mathbf{0}, \sigma_{1}^{2} \mathbf{X I}_{L} \mathbf{X}^{T}+\sigma^{2} \mathbf{I}_{L}\right)$.

\section{Compressive Sensing Based Bayesian Sparse Channel Estimation}

In this section, compressive sensing based Bayesian sparse channel estimation is proposed in two steps: (1) detect the position set of dominant channel taps and (2) then estimate sparse channel $\widetilde{\mathbf{h}}$ using MMSE algorithm. Obviously, how to find the dominant channel taps' position is a key technique with low-complexity Bayesian method for estimating sparse channels.

3.1. Position Detection on Dominant Channel Taps. According to the well-known Bayesian rules, the posterior of position indicator $\mathbf{g}$ can be written as

$$
P(\mathbf{g} \mid \mathbf{y})=\frac{P(\mathbf{y} \mid \mathbf{g}) P(\mathbf{g})}{\sum_{\mathbf{g} \prime \in G} P\left(\mathbf{y} \mid \mathbf{g}^{\prime}\right) P\left(\mathbf{g}^{\prime}\right)},
$$

where $G=\{0,1\}^{L}$ denotes all of possible position index sets of channel taps as shown in Figure 3. Equation (6) implies that estimating $\{P(\mathbf{g} \mid \mathbf{y})\}_{\mathbf{g} \in G}$ reduces to estimating $\{P(\mathbf{y} \mid \mathbf{g}) P(\mathbf{g})\}_{\mathbf{g} \in G}$. Due to the extremely computational complexity in (6), the huge size of $G$ makes it impractical to 
compute $P(\mathbf{g} \mid \mathbf{y})$ or $\left\{P\left(\mathbf{y} \mid \mathbf{g}^{\prime}\right) P\left(\mathbf{g}^{\prime}\right)\right\}$ for all $\mathbf{g}^{\prime} \in G$ in the case of high-dimensional broadband channels. By considering sparse structure in channels, only posteriors of dominant taps' position are needed for sparse channel estimation. Assuming that the set $G_{*}$ is responsible for position indicator of dominant channel taps, then the search space in $G_{*}$ rather than $G$ can be quite small and therefore practical to compute. Hence, the posteriors of dominant channel taps can be approximated by

$$
P(\mathbf{g} \mid \mathbf{y}) \approx \frac{P(\mathbf{y} \mid \mathbf{g}) P(\mathbf{g})}{\sum_{\mathbf{g}^{\prime} \in G_{*}} P\left(\mathbf{y} \mid \mathbf{g}^{\prime}\right) P\left(\mathbf{g}^{\prime}\right)},
$$

for dominant channel set $G_{*}$. Hence, exploiting the dominant channel taps set $G_{*}$ reduces to the search for $\mathbf{g} \in G_{*}$ which only computes the dominant values of $P(\mathbf{y} \mid \mathbf{g}) P(\mathbf{g})$ in (7). First of all, the probability density function (PDF) $P(\mathbf{y} \mid \mathbf{g})$ for position indicator $\mathbf{g} \in G_{*}$ can be written as

$$
P(\mathbf{y} \mid \mathbf{g})=\frac{1}{\sqrt{(2 \pi)^{L} \operatorname{det}(\mathbf{C}(\mathbf{g}))}} \exp \left(-\frac{1}{2} \mathbf{y}^{T} \mathbf{C}^{-1}(\mathbf{g}) \mathbf{y}\right)
$$

By transforming it in log-domain for convenience, then the position indicator (PI) $\mathrm{PI}(\mathbf{g}, \mathbf{y})$ can be given by

$$
\begin{aligned}
\mathrm{PI} & (\mathbf{g}, \mathbf{y}) \triangleq \ln P(\mathbf{y} \mid \mathbf{g}) P(\mathbf{g})=\ln P(\mathbf{y} \mid \mathbf{g})+\ln P(\mathbf{g}) \\
= & \ln P(\mathbf{y} \mid \mathbf{g})+\|\mathbf{g}\|_{0} \ln p_{1}+\left(L-\|\mathbf{g}\|_{0}\right) \ln \left(1-p_{1}\right) \\
= & -\frac{L}{2} \ln 2 \pi-\frac{1}{2} \operatorname{lndet}(\mathbf{C}(\mathbf{g}))-\frac{1}{2} \mathbf{y}^{T} \mathbf{C}^{-1}(\mathbf{g}) \mathbf{y} \\
& +\|\mathbf{g}\|_{0} \ln \frac{p_{1}}{1-p_{1}}+L \ln \left(1-p_{1}\right),
\end{aligned}
$$

which is a metric of position indicator $\mathbf{g}$. According to $\mathrm{PI}(\mathbf{g}, \mathbf{y})$ in (9), one can easily find that the position indicator depends on received signal, channel length, position indicator, and probability of nonzero taps. Due to the positive exponent relationship $P(\mathbf{g} \mid \mathbf{y})=e^{\mathrm{PI}(\mathbf{g}, \mathbf{y})}, \mathrm{PI}(\mathbf{g}, \mathbf{y})$ in (9) can also be considered as a measure function of $P(\mathbf{g} \mid \mathbf{y})$ on dominant channel taps. However, it is still unfeasible to get the position information of channel in practical system without considering channel estimation. According to [18], the mathematical expectation of $\operatorname{PI}(\mathbf{g}, \mathbf{y})$ can be given by

$$
\begin{aligned}
E\{\mathrm{PI}(\mathbf{g}, \mathbf{y})\}= & 2 N+L p_{1}\left(1-p_{1}\right) \\
& \times\left(\ln \left[\left(\frac{\sigma_{1}^{2}}{\sigma^{2}}+1\right) \frac{\left(1-p_{1}\right)}{p_{1}}\right]\right)^{2} .
\end{aligned}
$$

For a given pair $\left\{\mathbf{g}^{\prime}, \mathbf{y}\right\}, \operatorname{PI}\left(\mathbf{g}^{\prime}, \mathbf{y}\right)$ can be used to compare the mean $E\left\{\operatorname{PI}\left(\mathbf{g}^{\prime}, \mathbf{y}\right)\right\}$ and standard deviation $\sqrt{\operatorname{var}\left\{\operatorname{PI}\left(\mathbf{g}^{\prime}, \mathbf{y}\right)\right\}}$ in order to get a rough evaluation of $\left(\mathbf{g}^{\prime}, \mathbf{y}\right)$.

To reduce the search space in position set, we resort to an efficient method [13] to determine $G_{*}$ as follows. The basic idea is that the position set $\mathbf{g}$ of unknown channel yielding the dominant values of $P(\mathbf{g} \mid \mathbf{y})$ is equivalent to the high probability of $\operatorname{PI}(\mathbf{g}, \mathbf{y})$. The search starts with $\mathbf{g}=\mathbf{0}$ and the

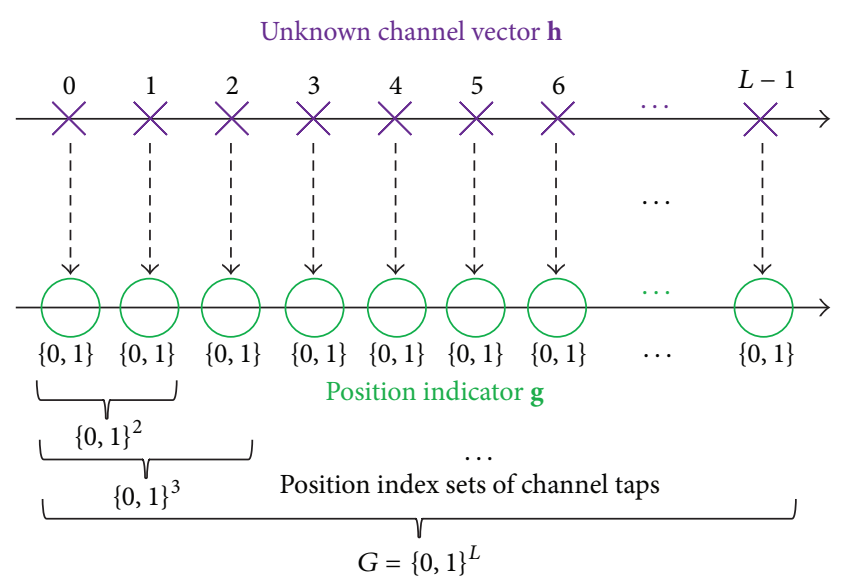

FIgURE 3: Graph illustration for all of possible position index sets of channel taps.

initial position set is set as $G^{(0)}$. If we change each element in $\mathbf{g}$, then it yields $L$ position indicators. Consider all of position indicators in a set and refer it to $G^{(1)}$. The metrics $\operatorname{PI}(\mathbf{g}, \mathbf{y})$ for the $L$ PI vectors in $G^{(1)}$ are then computed by (9), and elements of $G^{(1)}$ with the $D$ largest value of the dominant channel tap are collected in $G_{*}^{(1)}$. For each possible dominant taps' set in $G_{*}^{(1)}$, all positions of a second nonzero tap are then considered, yielding $\sum_{i=1}^{D}(L-i)=L D-D(D+1) / 2$ unique binary vectors to store in $G^{(2)}$. The $\mathrm{PI}(\mathrm{g}, \mathrm{y})$ for all possible vectors in $G^{(2)}$ are then computed, and the elements of $G^{(2)}$ with the $D$ largest value are collected in $G_{*}^{(2)}$. Then for each candidate vector in $G_{*}^{(2)}$, all possibilities of a third dominant channel tap are considered, and those with the $D$ largest channel taps are stored in $G_{*}^{(3)}$. The process continues until $G_{*}^{(S)}$ is computed, where $S$ can be chosen to make $\operatorname{Pr}\left(\|\mathbf{h}\|_{0}>S\right)$ sufficiently small to exploit all of channel sparsity. Note that $G_{*}^{(S)}$ constitutes the algorithm's final estimate of $G_{*}$ and later we denote $\widehat{G}_{*}$ as the final estimate. For better understanding of the PI update of dominant channel taps, an intuitive example is given in Figure 4, where the length of position indicator $\mathbf{g}$ is set as $L=5$; the number of largest value of $\mathrm{PI}$ is chosen as $D=1$, and the maximum number of nonzero taps is set as $S=3$.

For use with the aforementioned Bayesian matching pursuit (BMP) algorithm, we consider a fast metric update which computes the change in $\mathrm{PI}(\cdot)$ that results from the activation of a position of nonzero tap. More precisely, if we denote by $\mathbf{g}_{l}$ the vector identical to $\mathbf{g}$ except for the $l$ th coefficient, which is active in $\mathbf{g}_{l}$ but inactive in $\mathbf{g}$ (i.e., $\left[\mathbf{g}_{l}\right]_{l}=1$ and $[\mathbf{g}]_{l}=0$ ), then it is defined as

$$
d_{l}(\mathbf{g}) \triangleq \mathrm{PI}\left(\mathbf{g}_{l}, \mathbf{y}\right)-\mathrm{PI}(\mathbf{g}, \mathbf{y}),
$$

to track the change of active positions. Note that $\operatorname{PI}(\mathbf{g}, \mathbf{y})$ at the initial step is set as

$$
\operatorname{PI}(\mathbf{0}, \mathbf{y})=-\frac{L}{2} \ln 2 \pi-\frac{N}{2} \ln \sigma_{1}^{2}-\frac{1}{2 \sigma^{2}}\|\mathbf{y}\|_{2}^{2}+L \ln \left(1-p_{1}\right),
$$




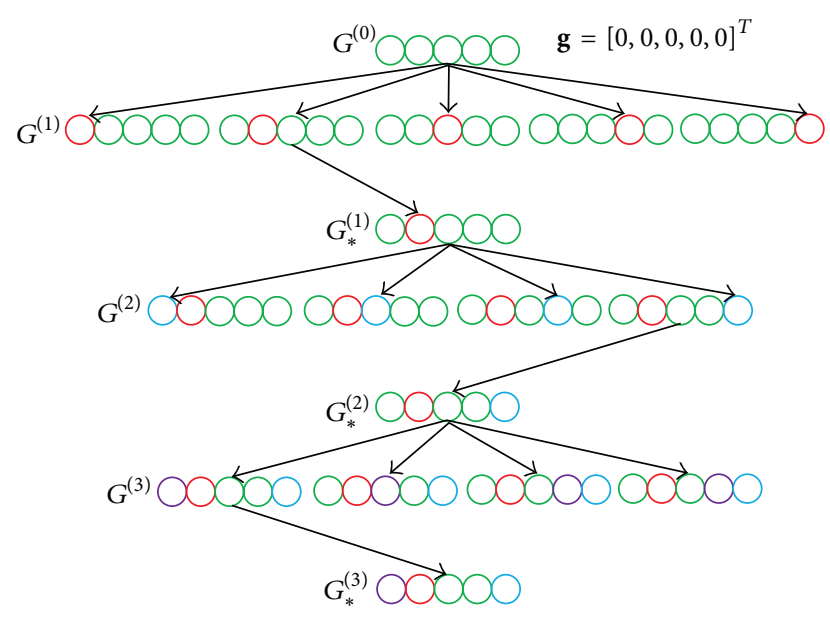

$\bigcirc 0$

FIgure 4: An intuitive example of position set selection on dominant channel taps, where the green circle denotes zero while the other colored circles denote one.

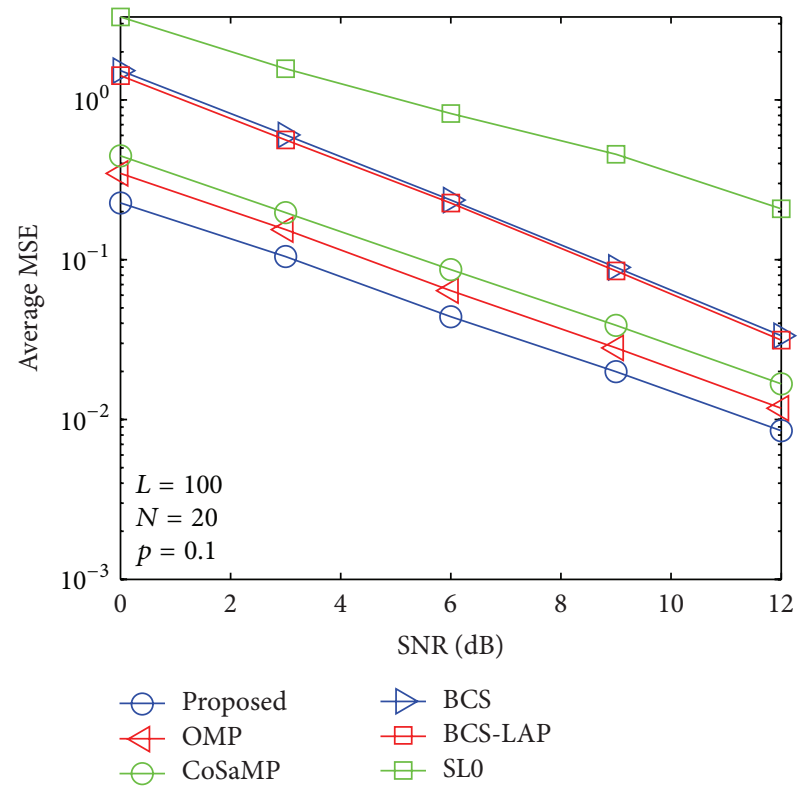

FIGURE 5: Average MSE performance versus SNR when $p_{1}=0.1$ and $N=20$.

via (9) and the fact that $\mathbf{C}(\mathbf{0})=\sigma_{1}^{2} \mathbf{I}_{L}$. To obtain the fast PI update, we start with the property that, for any $l$ and $\mathbf{g}$,

$$
\mathbf{C}\left(\mathbf{g}_{l}\right)=\mathbf{C}(\mathbf{g})+\sigma_{1}^{2} \mathbf{x}_{l} \mathbf{x}_{l}^{T},
$$

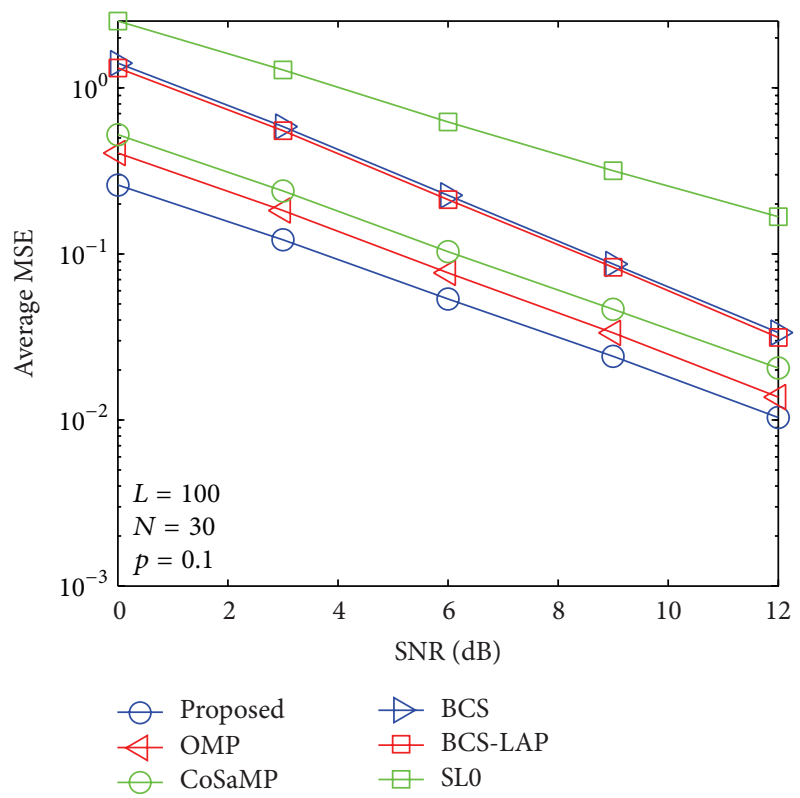

FIGURE 6: Average MSE performance versus SNR when $p_{1}=0.1$ and $N=30$.

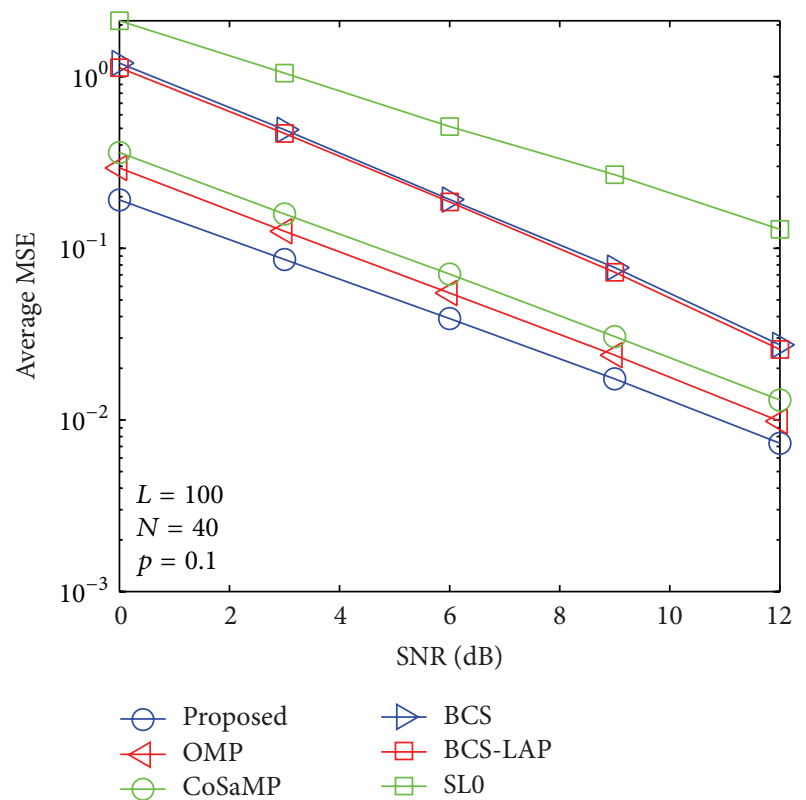

FIGURE 7: Average MSE performance versus SNR when $p_{1}=0.1$ and $N=40$.

for which the matrix inversion lemma implies

$$
\begin{gathered}
\mathbf{C}^{-1}\left(\mathbf{g}_{l}\right)=\mathbf{C}^{-1}(\mathbf{g})-\sigma_{1}^{2} \beta_{l} \mathbf{b}_{l} \mathbf{b}_{l}^{T}, \\
\mathbf{C}^{-1}(\mathbf{g})=\frac{1}{\sigma^{2}} \mathbf{I}_{N}-\sigma_{1}^{2} \sum_{i=1}^{p} \beta^{(i)} \mathbf{b}^{(i)}\left(\mathbf{b}^{(i)}\right)^{T} \\
\mathbf{b}_{l} \triangleq \mathbf{C}^{-1}(\mathbf{g}) \mathbf{x}_{l}=\frac{1}{\sigma^{2}} \mathbf{x}_{n}-\sigma_{1}^{2} \sum_{i=1}^{p} \beta^{(i)} \mathbf{b}^{(i)}\left(\mathbf{b}^{(i)}\right)^{T} \mathbf{x}_{l},
\end{gathered}
$$




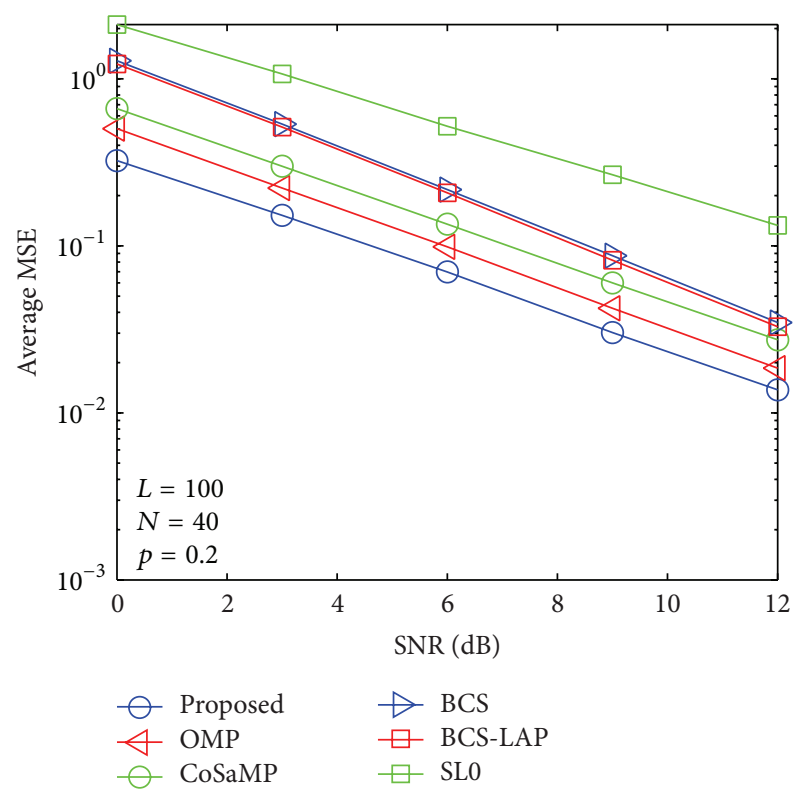

FIGURE 8: Average MSE performance versus SNR when $p_{1}=0.2$ and $N=40$.

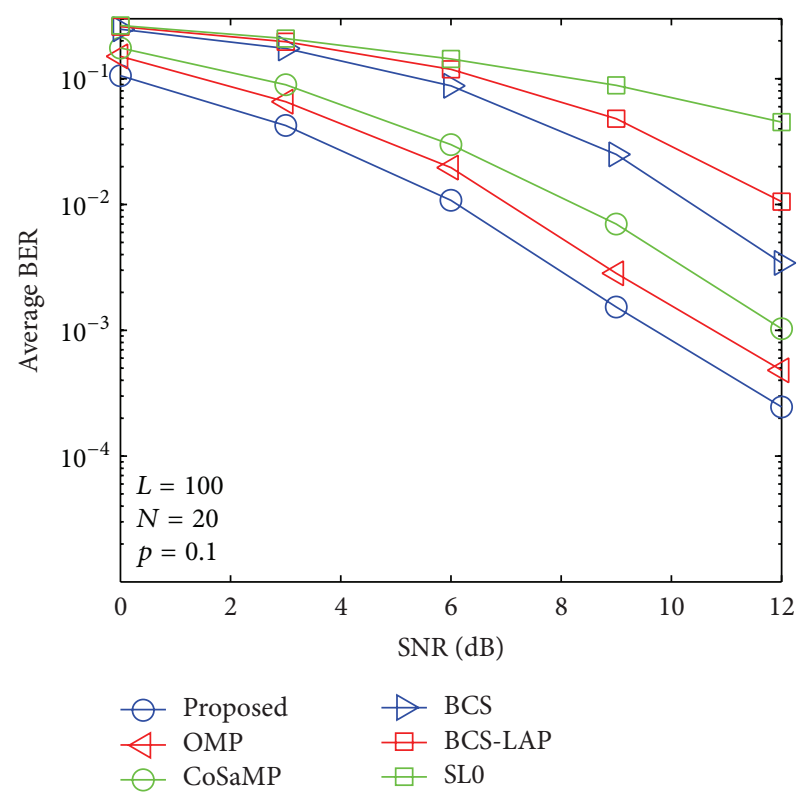

FIGURE 9: Average BER performance versus SNR when $p_{1}=0.1$ and $N=20$.

where $\mathbf{b}_{l}:=\mathbf{C}^{-1}(\mathbf{g}) \mathbf{x}_{l}$ and $\beta_{l}:=\left(1+\sigma_{1}^{2} \mathbf{x}_{l}^{T} \mathbf{b}_{l}\right)^{-1}$. Notice that the cost of computing $\beta_{l}$ in (14) is $\mathcal{O}\left(L N^{2}\right)$ if standard matrix

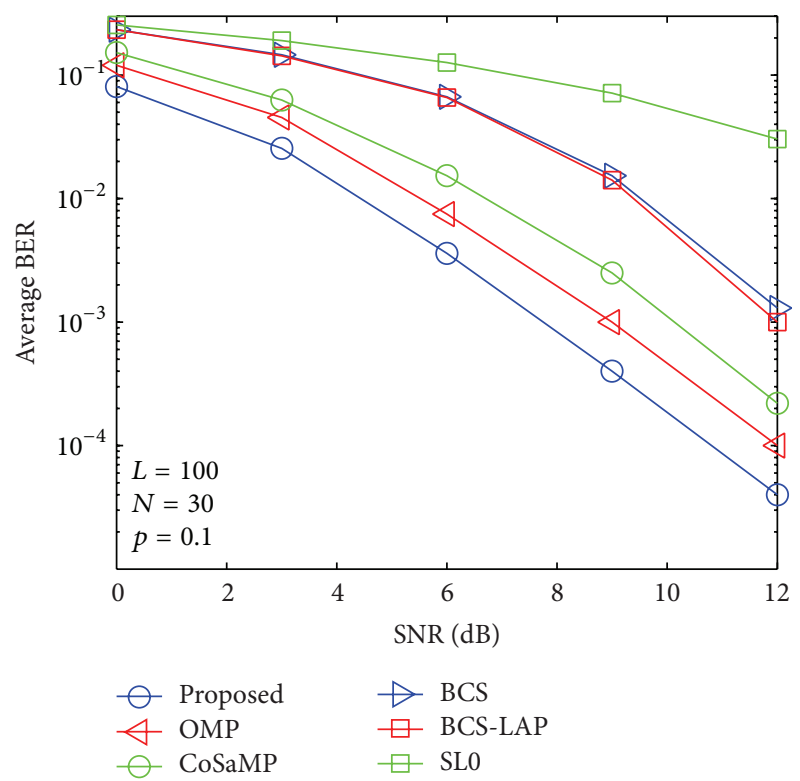

FIGURE 10: Average BER performance versus SNR when $p_{1}=0.1$ and $N=30$.

multiplication is used [13]. According to previous analysis, we can get

$$
\begin{aligned}
\mathbf{y}^{T} \mathbf{C}^{-1}\left(\mathbf{g}_{l}\right) \mathbf{y} & =\mathbf{y}^{T}\left(\mathbf{C}^{-1}(\mathbf{g})-\sigma_{1}^{2} \beta_{l} \mathbf{b}_{l} \mathbf{b}_{l}^{T}\right) \mathbf{y} \\
& =\mathbf{y}^{T} \mathbf{C}^{-1}(\mathbf{g}) \mathbf{y}-\sigma_{1}^{2} \beta_{l}\left(\mathbf{y}^{T} \mathbf{b}_{l}\right)^{2}, \\
\operatorname{lndet}\left(\mathbf{C}\left(\mathbf{g}_{l}\right)\right) & =\operatorname{lndet}\left(\mathbf{C}(\mathbf{g})+\sigma_{1}^{2} \mathbf{x}_{l} \mathbf{x}_{l}^{T}\right) \\
& =\ln \left[\left(1+\sigma_{1}^{2} \mathbf{x}_{l}^{T} \mathbf{C}^{-1}(\mathbf{g}) \mathbf{x}_{l}\right) \operatorname{det}(\mathbf{C}(\mathbf{g}))\right] \\
& =\operatorname{lndet}(\mathbf{C}(\mathbf{g}))-\ln \beta_{l}, \\
\left\|\mathbf{g}_{l}\right\|_{0} \ln \frac{p_{1}}{1-p_{1}} & =\left(\|\mathbf{g}\|_{0}+1\right) \ln \frac{p_{1}}{1-p_{1}} \\
& =\|\mathbf{g}\|_{0} \ln \frac{p_{1}}{1-p_{1}}+\ln \frac{p_{1}}{1-p_{1}},
\end{aligned}
$$

which, combined with (5), yield

$$
\mathrm{PI}\left(\mathbf{g}_{l}, \mathbf{y}\right)=\mathrm{PI}(\mathbf{g}, \mathbf{y})+\underbrace{\frac{1}{2} \ln \beta_{l}+\frac{\sigma_{1}^{2}}{2} \beta_{l}\left(\mathbf{y}^{T} \mathbf{b}_{l}\right)^{2}+\ln \frac{p_{1}}{1-p_{1}}}_{d_{l}(\mathbf{g})}
$$

In summary, $d_{l}(\mathbf{g})$ in (18) quantifies the change in $\mathrm{PI}(\cdot)$ due to the activation of the $l$ th position of $\mathbf{g}$.

Please note that the cost of computing $\left\{\beta_{l}\right\}_{l=0}^{L-1}$ via $\mathbf{b}_{l}:=$ $\mathbf{C}^{-1}(\mathbf{g}) \mathbf{x}_{l}$ and $\beta_{l}:=\left(1+\sigma_{1}^{2} \mathbf{x}_{l}^{T} \mathbf{b}_{l}\right)^{-1}$ is $\mathcal{O}\left(L N^{2}\right)$, if standard matrix multiplication is used. As we described, the complexity of this operation can be made linear in $N$ by exploiting the 


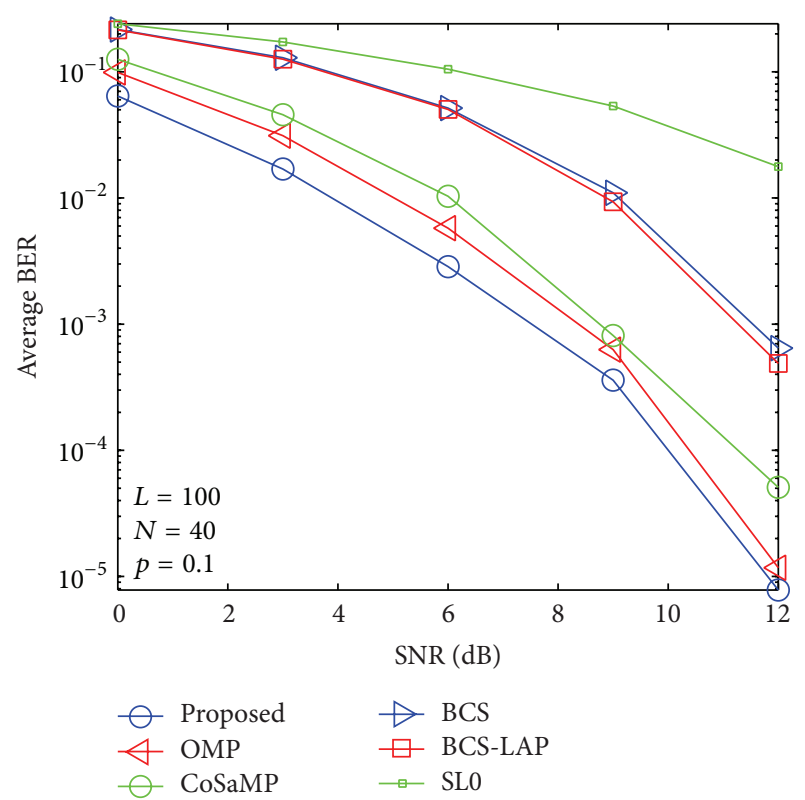

FIGURE 11: Average BER performance versus SNR when $p_{1}=0.1$ and $N=40$.

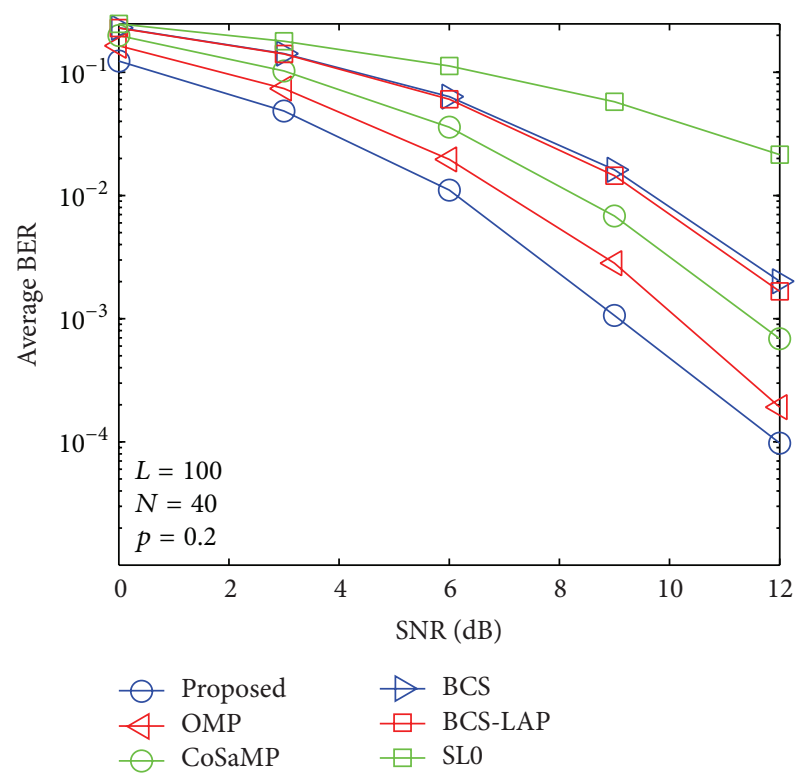

FIgURE 12: Average BER performance verses SNR when $p_{1}=0.2$ and $N=40$.

structure of $\mathbf{C}^{-\mathbf{1}}(\mathbf{g})$. Say that $\mathbf{t}=\left[t_{1}, t_{2}, \ldots, t_{p}\right]^{T}$ contains the indices of active elements in $\mathbf{g}$. Then from (14), we can get

$$
\mathbf{C}^{-1}(\mathbf{g})=\frac{1}{\sigma^{2}} \mathbf{I}_{N}-\sigma_{1}^{2} \sum_{i=1}^{p} \beta^{(i)} \mathbf{b}^{(i)} \underbrace{\mathbf{b}^{(i) T} \mathbf{x}_{l}}_{:=c_{l}^{(i)}}
$$

when activating the $l$ th position in $\mathbf{g}$. The key observation is that the coefficients $\left\{c_{l}^{(i)}\right\}_{l=0}^{L-1}$ need only to be computed once, that is, when index $t_{i}$ is active. Furthermore, $\left\{c_{l}^{(i)}\right\}_{l=0}^{L-1}$ only need to be computed for surviving indices $t_{i}$. According

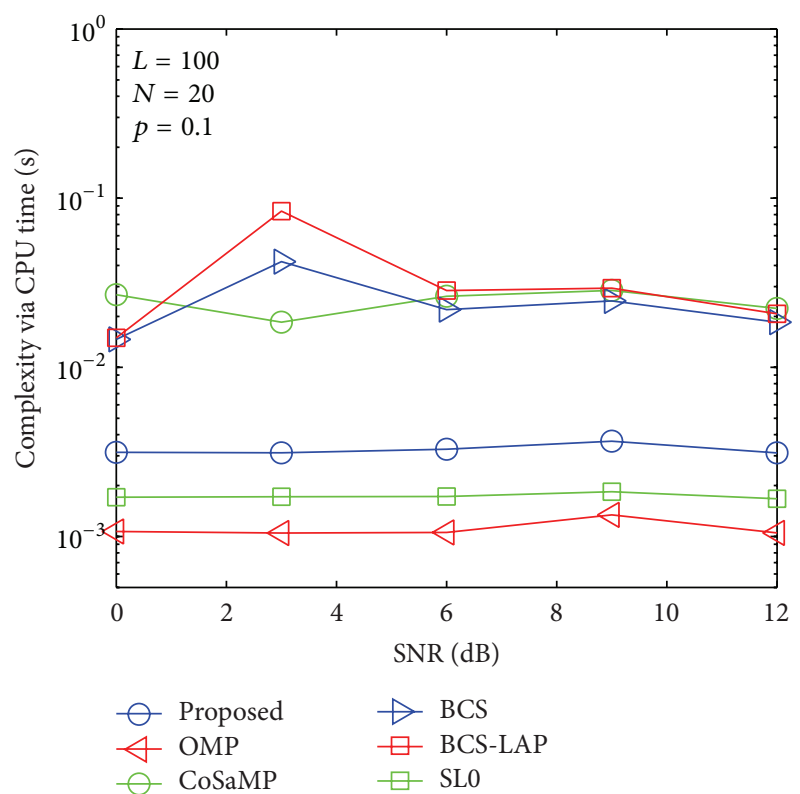

FIGURE 13: Computational complexity comparison via CPU time when $p_{1}=0.1$ and $N=20$.

to previous analysis in (20), the number of multiplications required by the algorithm is $\mathcal{O}(\mathrm{LNPD})$ [13]. Moreover, the complexity of the proposed algorithm could be reduced if the smaller $D$ is adopted.

3.2. MMSE for Estimating Values of Dominant Channel Taps. By utilizing the dominant taps' posteriors, the sparse channel can be estimated readily by MMSE algorithm as

$$
\begin{aligned}
\widetilde{\mathbf{h}} & =E\{\mathbf{h} \mid \mathbf{y}\} \\
& =\sum_{\mathbf{g} \in G} P(\mathbf{g} \mid \mathbf{y}) E\{\mathbf{h} \mid \mathbf{y}, \mathbf{g}\} \\
& \approx \sum_{\mathbf{g} \in G_{*}} P(\mathbf{g} \mid \mathbf{y}) E\{\mathbf{h} \mid \mathbf{y}, \mathbf{g}\} .
\end{aligned}
$$

According to the above introduction, compressive sensing based Bayesian sparse channel estimation could be implement by (20)-(22) with high estimation performance and low complexity.

\section{Computer Simulations}

In this section, the proposed BSCE estimator adopts 1000 independent Monte Carlo runs for averaging. The length of channel vector $\mathbf{h}$ is set as $N=100$. Values of dominant channel taps follow Gaussian distribution and their positions are randomly allocated within the length of $\mathbf{h}$ which is subjected to $E\left\{\|\mathbf{h}\|_{2}^{2}=1\right\}$. The received signal-to-noise ratio (SNR) is defined as $10 \log \left(E_{b} / \sigma_{n}^{2}\right)$, where $E_{b}=1$.

The proposed method is compared to five conventional sparse channel estimation methods using algorithms OMP [19], CoSaMP [9], BCS [14], BCS-LAP [15], and SL0 [20]. It 
TABLE 2: Simulation parameters.

\begin{tabular}{lcc}
\hline & Data modulation & BPSK \\
Number of subcarriers & $N_{d}=256$ \\
Transmitter Number of pilot symbols & $N \in\{20,30,40\}$ \\
& Length of CP & $N_{g}=16$ \\
& Pilot sequence & Random Gaussian sequence \\
\hline \multirow{2}{*}{ Channel } & Fading & Frequency-selective block \\
model & Prob. of nonzero taps & $L=100$ \\
& Power delay profile & Random Gaussian \\
\hline \multirow{2}{*}{ Receiver } & Channel estimation & BSCE \\
& Data detection & Zero forcing \\
\hline
\end{tabular}

was worth noting that these simulation parameters were chosen in accordance with detailed communication environment in this paper. The stopping error criteria threshold is set as $10^{-4}$ for all algorithms in Monte Carlo computer simulations. The initial noise variance for BSC and BSC-LAP is set as $\operatorname{var}(\mathbf{y}) / 10$, where $\operatorname{var}(\mathbf{y})=\left(1 /(N-1) \sum_{n=1}^{N}\left(y_{n}-\widehat{y}\right)\right)^{1 / 2}$ denotes standard derivation and $\hat{y}=1 / N \sum_{n=1}^{N} y_{i}$. In addition, the Laplace prior for BCS-LAP is computed automatically which was suggested in [15]. The parameters of FBMP algorithm were initialized as $\lambda_{1}=0.01, \mu_{1}=0, \sigma^{2}=0.05$, and $\sigma_{1}^{2}=2$. Computer simulation parameters are listed in Table 2.

4.1. MSE versus SNR. The estimation performance is evaluated by average mean square error (MSE) standard which is defined as

$$
\operatorname{MSE}\{\widetilde{\mathbf{h}}\}=E\|\mathbf{h}-\widetilde{\mathbf{h}}\|_{2}^{2}
$$

where $E\{\cdot\}$ denotes expectation operator and $\mathbf{h}$ and $\widehat{\mathbf{h}}$ are the actual channel vector and its channel estimator, respectively. In Figures 5, 6, 7, and 8, we compare the average MSE performance of the proposed channel estimator with traditional sparse channel estimators with respect to different channel sparseness, $p_{1}=0.1$ and $p_{1}=0.2$. As the four figures show, our proposed method can achieve better estimation performance than conventional methods. The lower bound is given by least square (LS) method (oracle) which utilized the channel position information. In this figure, it is easily found that the proposed method obtained lower MSE performance than conventional methods. In other words, if the proposed estimator is applied in data detection, smaller BER performance can be achieved when comparing with conventional methods.

4.2. BER versus SNR. By using the above channel estimators, signal transmission performances are evaluated as shown in Figures 9, 10, 11, and 12. From the four figures, average BER performance curves are depicted with respect to SNR for binary phase shift keying (BPSK) data. We can see that the BER performance of the proposed method is more close to lower bound which is given by ideal channel estimator whose nonzero taps' positions are known. Here, only low signal

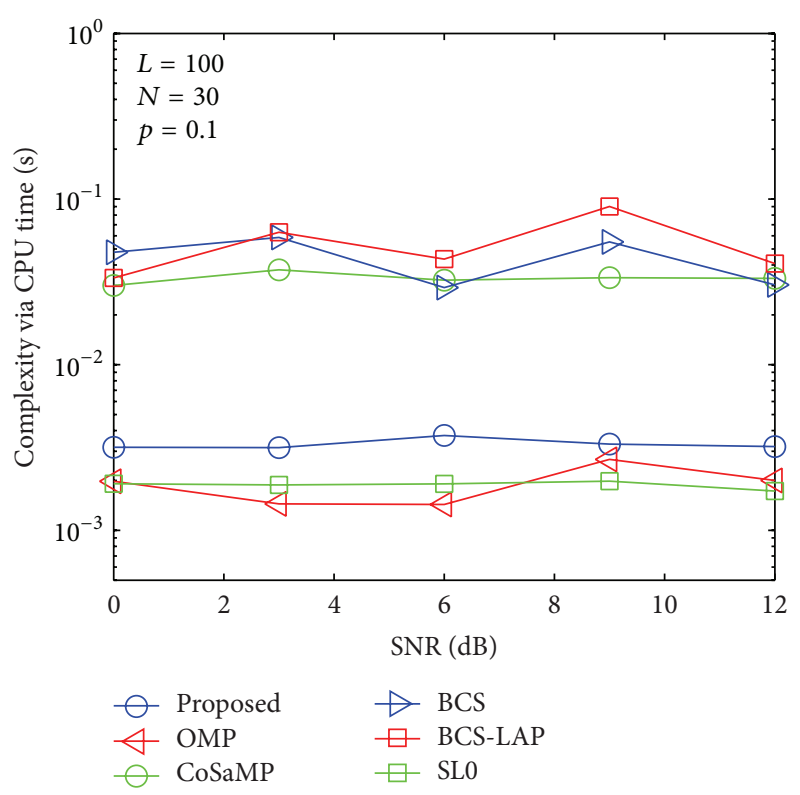

FIGURE 14: Computational complexity comparison via CPU time when $p_{1}=0.1$ and $N=30$.

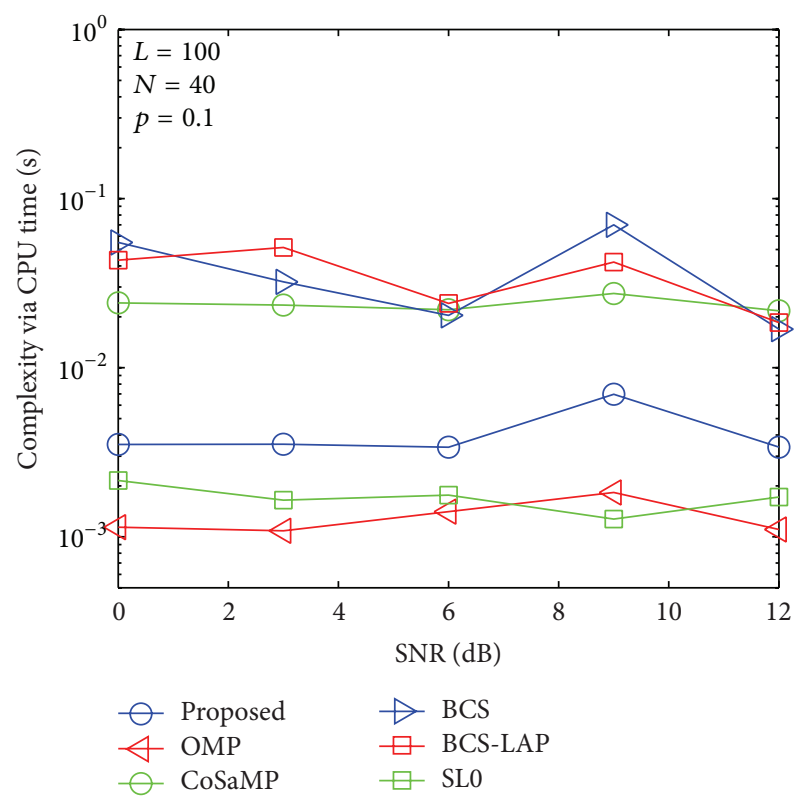

FIGURE 15: Computational complexity comparison via CPU time when $p_{1}=0.1$ and $N=40$.

modulation was considered for BER evaluation. It is very easy to predict that our proposed method could improve BER performance in case of high signal modulation.

4.3. Complexity Evaluation. To compare the computational complexity of the proposed method with other methods, CPU time is adopted for evaluation standard as shown in Figures 13, 14, 15, and 16. It is worth mentioning that although the CPU time is not an exact measure of complexity, it can give us a rough estimation of computational complexity. Our 


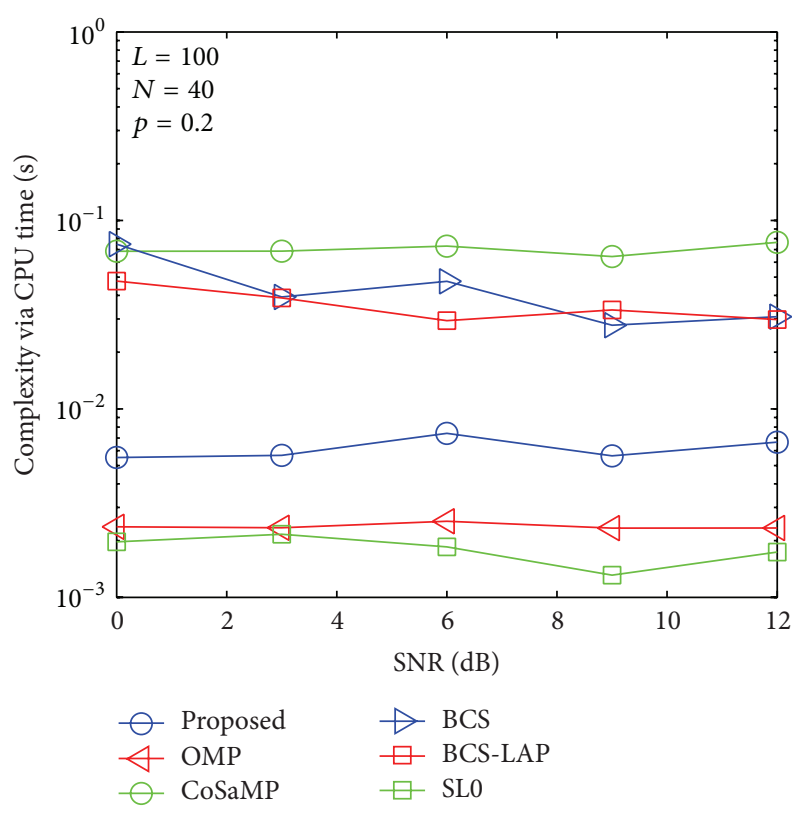

FIgURE 16: Computational complexity comparison via CPU time when $p_{1}=0.2$ and $N=40$.

simulations are performed in MATLAB 2012 environment using a $2.90 \mathrm{GHz}$ Intel i7 processor with $8 \mathrm{~GB}$ of memory and under Microsoft Windows 8 (64 bit) operating system. For comprehensive comparing between our proposed method and other methods in different length of training signal and different channel sparsity, we simulate their comparison results in Figures 13-16. As the four figures shown, the complexity of the proposed method is close to OMP and SL0based methods and lower than CoSaMP, BCS, and BCS-LAP based methods. It is well known that the complexity of OMP and SL0 is very low on sparse channel estimation [10, 22]. Hence, comparing with traditional methods, our proposed method can achieve better estimation performance and low complexity.

\section{Conclusion}

Traditional sparse channel estimation methods are vulnerable to noise and column coherence interference in training matrix. Their primary aim is an attempt to exploit sparse structure information without a report of posterior channel uncertainty. To improve the estimation performance, fast Bayesian matching pursuit algorithm with application to sparse channel estimation has not only exploited the channel sparsity but also mitigated the unexpected inferences in training matrix. In addition, the proposed method has revealed potential ambiguity among multiple channel estimators that are ambiguous due to observation of noise or correlation among columns in the training signal. Computer simulation results have showed that proposed method improved the estimation performance with comparable computational complexity when comparing with traditional methods.

\section{Conflict of Interests}

The authors declare that there is no conflict of interests regarding the publication of this paper. The authors of the paper do not have a direct financial relation that might lead to a conflict of interests for any of the authors.

\section{References}

[1] L. Dai, Z. Wang, and Z. Yang, "Compressive sensing based time domain synchronous OFDM transmission for vehicular communications," IEEE Journal on Selected Areas in Communications, vol. 31, no. 9, pp. 460-469, 2013.

[2] L. Dai, Z. Wang, and Z. Yang, "Next-generation digital television terrestrial broadcasting systems: key technologies and research trends," IEEE Communications Magazine, vol. 50, no. 6, pp. 150-158, 2012.

[3] N. Czink, X. Yin, H. Özcelik, M. Herdin, E. Bonek, and B. H. Fleury, "Cluster characteristics in a MIMO indoor propagation environment," IEEE Transactions on Wireless Communications, vol. 6, no. 4, pp. 1465-1475, 2007.

[4] E. J. Candès, J. Romberg, and T. Tao, "Robust uncertainty principles: exact signal reconstruction from highly incomplete frequency information," IEEE Transactions on Information Theory, vol. 52, no. 2, pp. 489-509, 2006.

[5] D. L. Donoho, "Compressed sensing," IEEE Transactions on Information Theory, vol. 52, no. 4, pp. 1289-1306, 2006.

[6] G. Z. Karabulut and A. Yongaçoglu, "Sparse channel estimation using orthogonal matching pursuit algorithm," in Proceedings of the IEEE 60th Vehicular Technology Conference (VTC '04), pp. 3880-3884, Los Angeles, Calif, USA, September 2004.

[7] G. Tauböck, F. Hlawatsch, D. Eiwen, and H. Rauhut, "Compressive estimation of doubly selective channels in multicarrier systems: leakage effects and sparsity-enhancing processing," IEEE Journal on Selected Topics in Signal Processing, vol. 4, no. 2, pp. 255-271, 2010.

[8] G. Gui, Q. Wan, W. Peng, and F. Adachi, "Sparse multipath channel estimation using compressive sampling matching pursuit algorithm," in Proceedings of the 7th IEEE VTS Asia Pacific Wireless Communications Symposium (APWCS '10), pp. 10-14, Kaohsiung, Taiwan, May 2010.

[9] D. Needell and J. A. Tropp, "CoSaMP: iterative signal recovery from incomplete and inaccurate samples," Applied and Computational Harmonic Analysis, vol. 26, no. 3, pp. 301-321, 2009.

[10] G. Gui, Q. Wan, and W. Peng, "Fast compressed sensingbased sparse multipath channel estimation with smooth L0 algorithm," in Proceedings of the 3rd International Conference on Communications and Mobile Computing (CMC'11), pp. 242245, Qingdao, China, April 2011.

[11] G. H. Mohimani, M. Babaie-Zadeh, and C. Jutten, "Complexvalued sparse representation based on smoothed $\ell^{0}$ norm," in Proceedings of the IEEE International Conference on Acoustics, Speech and Signal Processing (ICASSP '08), pp. 3881-3884, April 2008.

[12] W. U. Bajwa, J. Haupt, A. M. Sayeed, and R. Nowak, "Compressed channel sensing: a new approach to estimating sparse multipath channels," Proceedings of the IEEE, vol. 98, no. 6, pp. 1058-1076, 2010.

[13] P. Schniter, L. C. Potter, and J. Ziniel, "Fast bayesian matching pursuit," in Proceedings of the Information Theory and Applications Workshop (ITA '08), pp. 326-333, San Diego, Calif, USA, February 2008. 
[14] S. Ji, Y. Xue, and L. Carin, "Bayesian compressive sensing," IEEE Transactions on Signal Processing, vol. 56, no. 6, pp. 2346-2356, 2008.

[15] S. D. Babacan, R. Molina, and A. K. Katsaggelos, "Bayesian compressive sensing using laplace priors," IEEE Transactions on Image Processing, vol. 19, no. 1, pp. 53-63, 2010.

[16] C. Carbonelli and U. Mitra, "Clustered channel estimation for UWB multiple antenna systems," IEEE Transactions on Wireless Communications, vol. 6, no. 3, pp. 970-981, 2007.

[17] L. Dai, Z. Wang, and Z. Yang, "Spectrally efficient timefrequency training OFDM for mobile large-scale MIMO systems," IEEE Journal on Selected Areas in Communications, vol. 31, no. 2, pp. 251-263, 2013.

[18] P. Schniter, L. C. Potter, and J. Ziniel, "Fast Bayesian matching pursuit: model uncertainty and parameter estimation for sparse linear models," OSU ECE Technical Report, 2009.

[19] J. A. Tropp and A. C. Gilbert, "Signal recovery from random measurements via orthogonal matching pursuit," IEEE Transactions on Information Theory, vol. 53, no. 12, pp. 4655-4666, 2007.

[20] H. Mohimani, M. Babaie-Zadeh, and C. Jutten, "A fast approach for overcomplete sparse decomposition based on smoothed $\ell^{0}$ norm," IEEE Transactions on Signal Processing, vol. 57, no. 1, pp. 289-301, 2009.

[21] F. Adachi, D. Garg, S. Takaoka, and K. Takeda, "Broadband CDMA techniques," IEEE Wireless Communications, vol. 12, no. 2, pp. 8-18, 2005.

[22] G. Gui, A. Mehbodniya, Q. Wan, and F. Adachi, "Sparse signal recovery with OMP algorithm using sensing measurement matrix," IEICE Electronics Express, vol. 8, no. 5, pp. 285-290, 2011. 

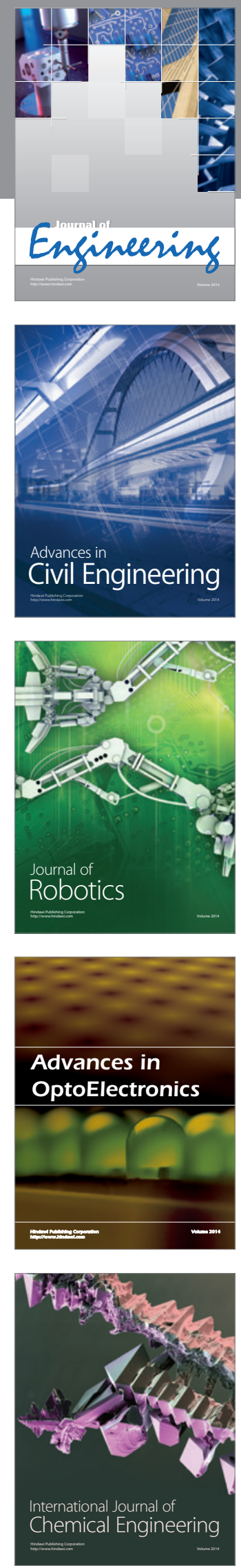

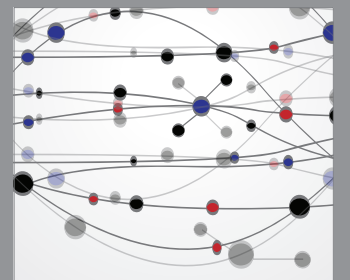

The Scientific World Journal
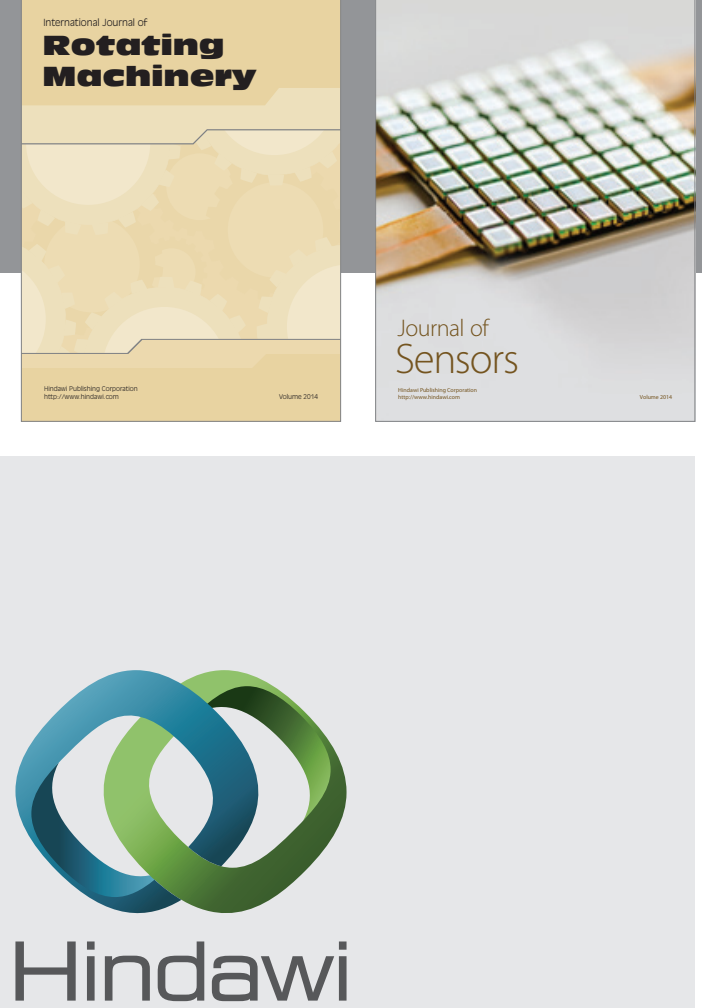

Submit your manuscripts at http://www.hindawi.com
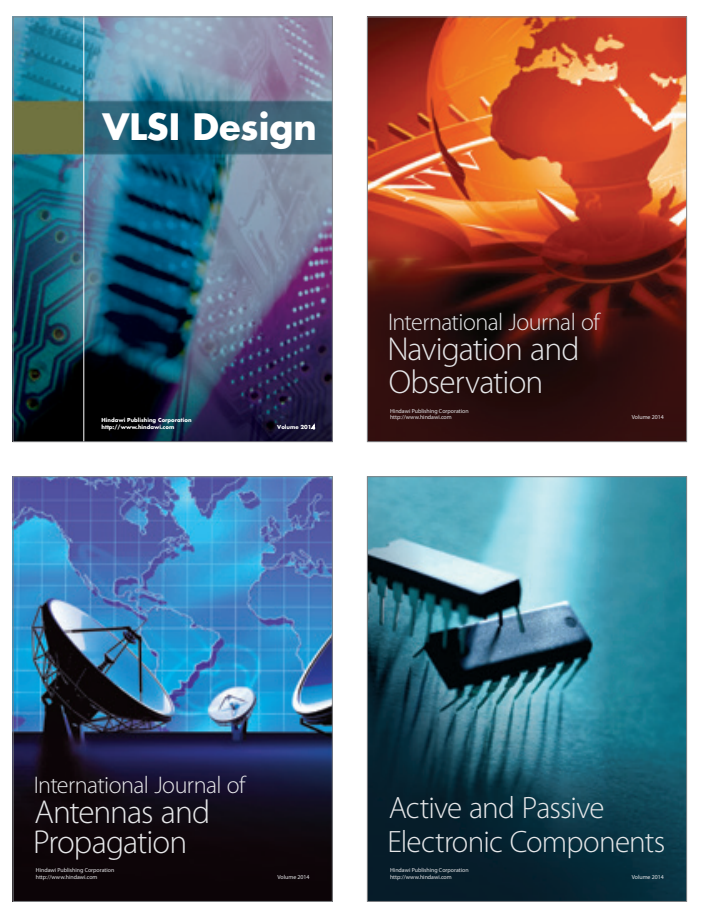
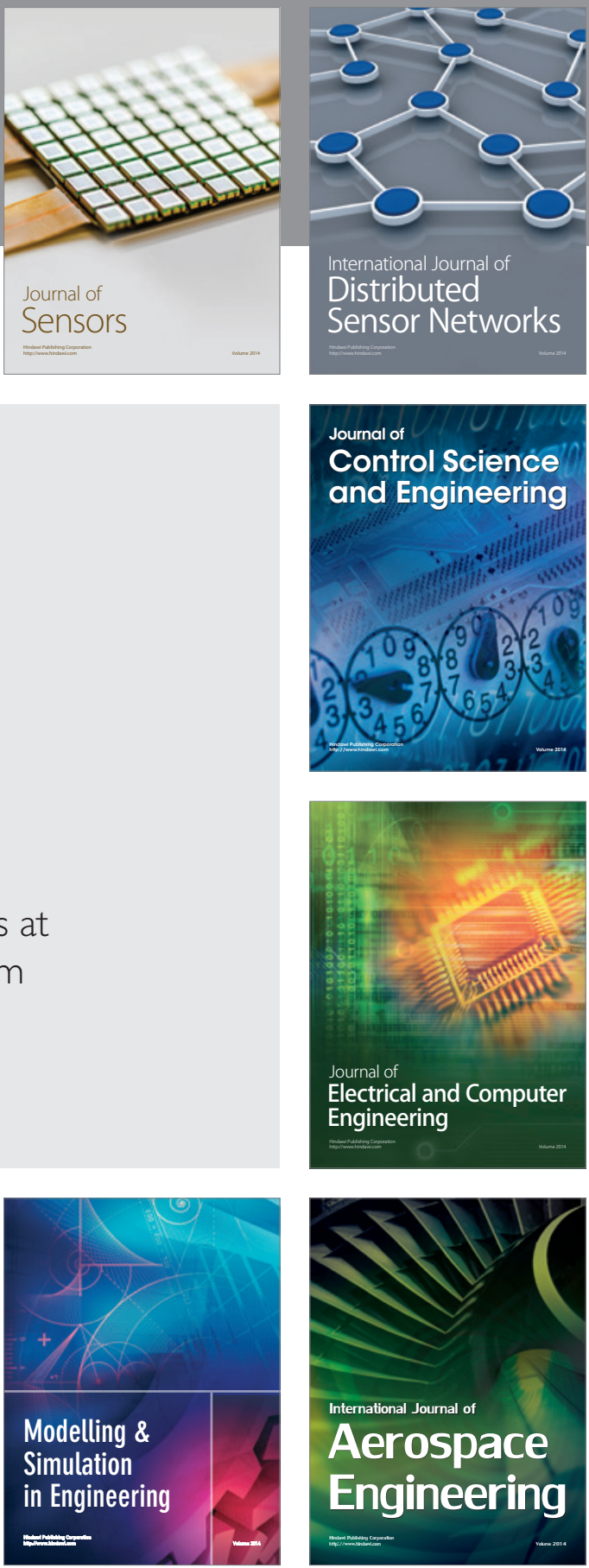

Journal of

Control Science

and Engineering
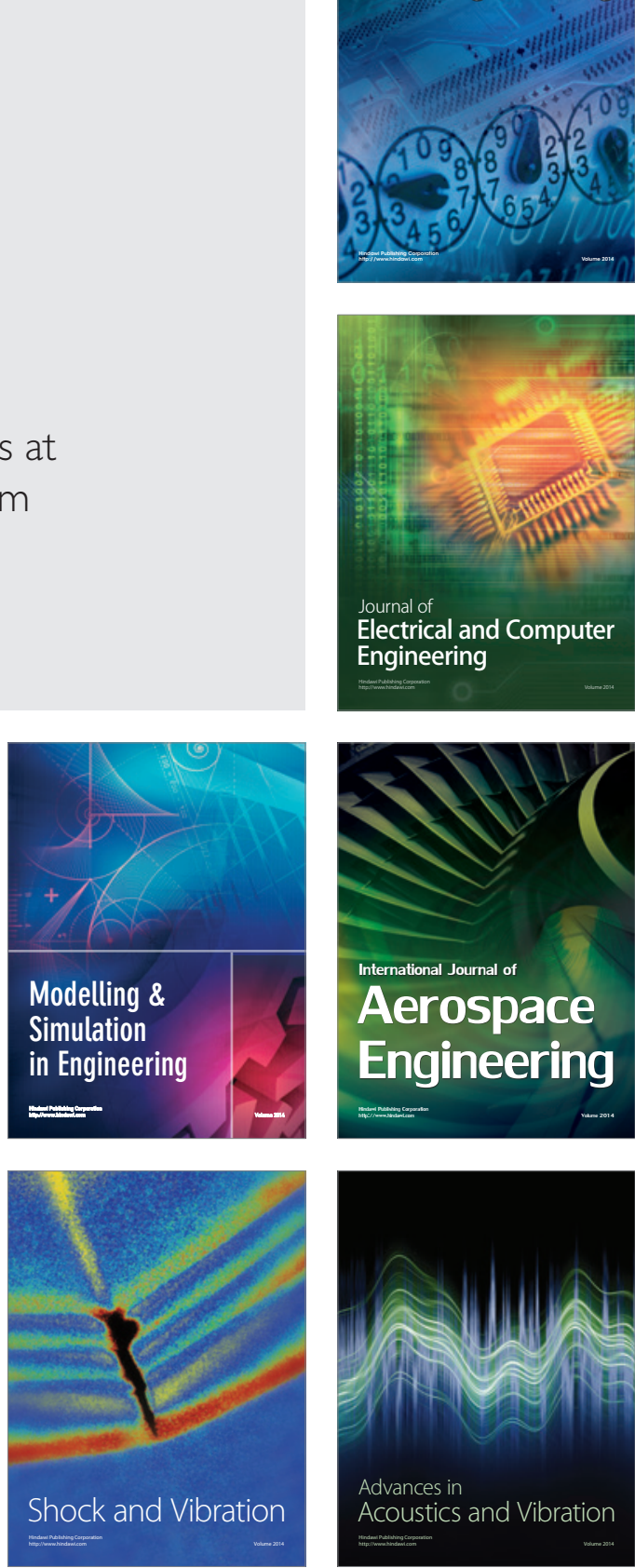